\title{
MAPPING BETWEEN NONLINEAR SCHRÖDINGER EQUATIONS WITH REAL AND COMPLEX POTENTIALS
}

\author{
MARIO SALERNO
}

Communicated by Andrei Ludu

\begin{abstract}
A mapping between the stationary solutions of nonlinear Schrödinger equations with real and complex potentials is constructed and a set of exact solutions with real energies are obtained for a large class of complex potentials. As specific examples we consider the case of dissipative periodic soliton solutions of the nonlinear Schrödinger equation with complex potential.
\end{abstract}

\section{Introduction}

Nonlinear wave phenomena with time evolutions governed by non hermitian Hamiltonians are presently attracting a great interest both from the theoretical and the applicative point of view. The non hermiticity is in general due to the presence of a complex potential in the Hamiltonian accounting for typical dissipative and amplification effects met in classical and quantum contexts [5, 12]. In particular, dissipative solitons [4] of the nonlinear Schrödinger (NLS) equation with periodic complex potentials have been extensively investigated during the past years in connections with the propagation of light in nonlinear optical fibers with periodic modulations of the complex refractive index $[13,18]$. Recently similar studies were done for matter wave solitons of Bose-Einstein condensates (BEC) trapped in absorbing optical lattices $[1,7]$ and in the presence of three body interatomic interactions [3]. In the linear context, the recent discovery [6] that the Schrödinger eigenvalue problem with complex potentials that are invariant under the combined parity and time reversal symmetry (so called $\mathcal{P} \mathcal{T}$-potentials), may have fully real spectrum, has raised interest also in view of possible connection with the theory of quantum dissipative systems [10]. Complex potentials with $\mathcal{P} \mathcal{T}$-symmetry are presently investigated in nonlinear optics [11] where it has been demonstrated that nonlinear media with linear damping and amplifications that are $\mathcal{P} \mathcal{T}$-symmetric can support stable stationary localized and periodic states [14]. Also, quite recently, physical systems with $\mathcal{P} \mathcal{T}$-symmetry have been successfully implemented in real experiments $[9,15,17]$. Solutions of the NLS equation with a complex potential which belong to the real part of the spectrum (real energies or real chemical 
potentials) can exist, however, for generic complex potentials and it is therefore of interest to characterize them in general, independently from the $\mathcal{P} \mathcal{T}$-symmetry.

The aim of the present paper is to show how one can systematically construct stationary solutions of the complex nonlinear Schrödinger equation via a mapping between real and complex NLS equations. The problem is formulated in terms of a nonlocal eigenvalue problem which involves only real potentials, whose eigenfunctions and eigenvalues fix amplitudes and energies of the stationary solutions of the complex NLS equation, respectively. The complex potentials and the phases of the solutions are also determined self-consistently through the mapping. To illustrate our approach we discuss the case of the NLS equation with different complex potentials for which we construct periodic dissipative solitons in the form of elliptic functions.

The paper is organized as follows. In Section 2 we introduce model equations and illustrate the mapping used to determine the solutions. In Section 3 we show how to construct exact solutions of the NLS with periodic complex potentials while in the last section the main results of the paper will be briefly summarized.

\section{Model Equations and Mapping}

The model equation we consider is the NLS equation with real and complex potentials both of linear and nonlinear types, e.g.

$$
\mathrm{i} \psi_{t}=-\frac{1}{2} \psi_{x x}+\left(V_{l}(x)+\mathrm{i} W_{l}(x)\right) \psi+\left(\sigma+V_{n l}(x)+\mathrm{i} W_{n l}(x)\right)|\psi|^{2} \psi .
$$

The case of the linear Schrodinger equation (e.g. $\sigma=V_{n l}=W_{n l}=0$ ) can be used as an example of quantum dissipative system. In the nonlinear case the above equation can appear in connection with several interesting phenomena including light propagation in photonic crystals and Bose-Einstein condensates. Due to the possibility of different physical applications we shall keep equation (1) in normalized form, looking for stationary solutions of the type

$$
\psi(x, t)=A(x) \mathrm{e}^{\mathrm{i} \theta(x)} \mathrm{e}^{-\mathrm{i} \omega t}
$$

with the amplitude $A(x)$ and phase $\theta(x)$ as real functions. Substituting this expression into equation(1), we obtain the system of equations

$$
\begin{aligned}
\omega A+\frac{1}{2} A_{x x}-\sigma A^{3}-\frac{A}{2}\left(\theta_{x}\right)^{2}-V_{l} A-V_{n l} A^{3} & =0 \\
\frac{1}{2} A \theta_{x x}+A_{x} \theta_{x}-W_{l} A-W_{n l} A^{3} & =0 .
\end{aligned}
$$


These equations can be easily separated. In this respect notice that by multiplying equation (4) by $A$ and integrating it twice one obtains

$$
\frac{1}{2} \theta(x)=B_{2}+\int_{-\infty}^{x} \frac{B_{1}+F(y)}{A^{2}(y)} \mathrm{d} y
$$

with

$$
F(y)=\int_{-\infty}^{y}\left[W_{l}(z)+W_{n l}(z) A^{2}(z)\right] A^{2}(z) \mathrm{d} z
$$

and $B_{1}, B_{2}$ integration constants. By substituting equation (5) into equation (3) we obtain the following nonlinear eigenvalue problem for the real amplitude $A$

$$
\left\{-\frac{1}{2} \frac{\partial}{\partial x^{2}}+V_{l}+\left(\sigma+V_{n l}\right) A^{2}+2\left(\frac{F(x)}{A^{2}}\right)^{2}\right\} A=\omega A
$$

where the integration constants $B_{1}, B_{2}$, have been fixed to zero for simplicity. Note that for stationary solutions equation (7) is completely equivalent to equation (1) in the sense that any solution of (7) gives a stationary solution of (1) with the phase fixed by (5). Also note that the dependence on the complex potentials in the eigenvalue problems comes through the function $F$ and for an arbitrary $F(x)$ (e.g. arbitrary complex potentials) the problem can become singular. It is possible, however, to construct potentials $W_{l}$ and $W_{n l}$ (e.g. functions $F$ ) so that the solutions of (7) are regular. This establishes a mapping between stationary solutions of the NLS equation with real potentials and stationary solution of equation (1) with the phase given by (5). In this respect, one can take $F$ in general to be an analytical function of $A^{2}$ and derivatives e.g. $F(x) \equiv F\left(A^{2},\left(A^{2}\right)_{x}, \ldots\right)$. In the simplest case $F$ can be taken of the form

$$
F(x)=\frac{1}{2} C_{n} A^{n+2}, \quad n=0,1,2 \ldots
$$

with $C_{n}$ constants to be determined. Equation (7) then reduces to the following NLS real eigenvalue problem

$$
\left\{-\frac{1}{2} \frac{\partial}{\partial x^{2}}+V_{l}+\left(\sigma+V_{n l}\right) A^{2}+\frac{C_{n}^{2}}{2} A^{2 n}\right\} A=\omega A
$$

which can be solved analytically for particular forms of the potentials $V_{l}, V_{n l}$, or numerically with high accuracy (using for example the self-consistent method discussed in [16]) for generic real potentials. In the following we therefore assume that the real amplitudes $A$ and frequencies $\omega$ for given $V_{l}$ and $V_{n l}$ are exactly obtained from (9), either analytically or numerically. 
On the other hand from equation (8) one can characterize the complex potentials which support such solutions. Using equation (6) we have indeed that equation (8) is satisfied if the amplitude $A$ is related to $W_{l}$ and $W_{n l}$ by the relation

$$
W_{l}+W_{n l} A^{2}=C_{n}\left(\frac{n+2}{2 n}\right) \frac{\mathrm{d} A^{n}}{\mathrm{~d} x}
$$

and from equations (5), (8), one gets that the phase is given by

$$
\theta(x)=C_{n} \int_{-\infty}^{x} A^{n} \mathrm{~d} y .
$$

Note that in this case equation (10) allows to relate the constant $C_{n}$ to the amplitude of the solution, $A_{0}$, and the amplitudes $W_{0 l}, W_{0 n l}$, of the linear and nonlinear complex potentials, respectively. In particular, for the case $W_{n l}=0$ we have that

$$
C_{n}=\frac{2}{n+2} \frac{W_{0 l}}{A_{0}^{n}}, \quad W_{0 n l}=0
$$

while for $W_{l}=0$ one obtains

$$
C_{n}=\frac{2}{n+2} \frac{W_{0 n l}}{A_{0}^{n-2}}, \quad W_{0 l}=0 .
$$

It is worth to note that while the case $n=1$ leads to a pure cubic NLS eigenvalue problem, the case $n>1$ introduces higher order nonlinearities in equation (9) which can however be eliminated by redefying the linear real potential as

$$
V_{l}=\tilde{V}_{l}-\frac{C_{n}^{2}}{2} A^{2 n}
$$

or the nonlinear real potential as

$$
V_{n l}=\tilde{V}_{n l}-\frac{C_{n}^{2}}{2} A^{2 n-2}
$$

(or a combination of both). Also notice that equations (2), (10) - (13) allow to map solutions of the real eigenvalue problem (9) into solutions of the NLS equation (1) with the corresponding complex potentials determined as in (10). It is clear that this approach can be extended to functions of the type

$$
F(x)=\frac{1}{2} \sum_{n=0}^{k} C_{n} A^{n+2}, \quad k=0,1,2 \ldots
$$


In this case coefficient $C_{n}$ are self-consistently determined from the real eigenvalue problem

$$
\left\{-\frac{1}{2} \frac{\partial}{\partial x^{2}}+V_{l}+\left(\sigma+V_{n l}\right) A^{2}+\frac{1}{2}\left(\sum_{n} C_{n} A^{n}\right)^{2}\right\} A=\omega A
$$

and complex potentials and phase are given by

$$
\begin{gathered}
W_{l}+W_{n l} A^{2}=\frac{1}{A^{2}} \frac{\mathrm{d} F}{\mathrm{~d} x} \\
\theta(x)=\sum_{n} C_{n} \int_{-\infty}^{x} A^{n} \mathrm{~d} y .
\end{gathered}
$$

Note that the sum in equation (16) can include infinite terms and to have a map between real and complex NLS equations it is necessary to subtract higher order nonlinearities from the real linear and nonlinear potentials as done in equations (14)-(15). Finally we remark that if the functions $A_{x} / A, A_{x x} / A, \ldots$ are bounded, the expression (16) can be further generalized as

$$
F(x)=\sum_{n, m}^{k} C_{n, m} \frac{\mathrm{d}^{m} A^{n+2}}{\mathrm{~d} x^{m}}
$$

with $C_{n, m}$ suitable constants and with the complex potentials determined as (18). In all these cases a map between solutions of the real eigenvalue problem (17) and solutions of the NLS equation (1) is constructed.

The mapping guarantees that the constructed solutions always have real energies and may be therefore of physical interest. We finally remark that a similar approach based on a priori fixing of the solution and a posteriori determination of the complex potential, has been considered also in $[2,8]$, although not in terms of a mapping between stationary solutions of NLS equations. In the following we illustrate how the mapping works on some specific example.

\section{Nonlinear Schrödinger Equation with Complex Potentials}

\subsection{Case $n=1$}

Let us consider first the simplest ansatz (8) with $n=1$. We fix the nonlinearity to be attractive $(\sigma<0)$ and restrict to linear complex potentials (i.e., $W_{n l}=V_{n l}=0$ ) 
and with linear potential of the form $V_{l}=V_{0 l} \mathrm{cn}^{2}(x, k)$. In this case the real eigenvalue problem (9)

$$
\left[-\frac{1}{2} \frac{\partial}{\partial x^{2}}+V_{0 l} \mathrm{cn}(x, k)^{2}+\left(\sigma+\frac{C_{1}^{2}}{2}\right) A^{2}\right] A=\omega A
$$

admits the following exact solutions in terms of elliptic functions

a) $A(x)=A_{0} \operatorname{cn}(x, k)$

$$
A_{0}= \pm \sqrt{\frac{2\left(k^{2}+V_{0 l}\right)}{2|\sigma|-C_{1}^{2}}}, \quad \omega=\frac{1-2 k^{2}}{2}
$$

b) $A(x)=A_{0} \operatorname{sn}(x, k)$

$$
A_{0}= \pm \sqrt{\frac{2\left(k^{2}+V_{0 l}\right)}{C_{1}^{2}-2|\sigma|}}, \quad \omega=\frac{1+k^{2}}{2}+V_{0 l}
$$

c) $A(x)=A_{0} \operatorname{dn}(x, k)$

$$
A_{0}= \pm \frac{1}{k} \sqrt{\frac{2\left(k^{2}+V_{0 l}\right)}{2|\sigma|-C_{1}^{2}}}, \quad \omega=\frac{k^{2}}{2}-1+V_{0 l}\left(1-\frac{1}{k^{2}}\right) \text {. }
$$

Similar solutions can be constructed for the case of a repulsive nonlinearity $\sigma>0$ with linear potentials of the form $V_{l}=V_{0 l} \operatorname{sn}^{2}(x, k)$. In this case we have

d) $\quad A(x)=A_{0} \operatorname{cn}(x, k)$

$$
A_{0}= \pm \sqrt{\frac{2\left(V_{0 l}-k^{2}\right)}{C_{1}^{2}+2 \sigma}}, \quad \omega=\frac{1-2 k^{2}}{2}+V_{0 l}
$$

e) $A(x)=A_{0} \operatorname{sn}(x, k)$

$$
A_{0}= \pm \sqrt{\frac{2\left(k^{2}-V_{0 l}\right)}{C_{1}^{2}+2 \sigma}}, \quad \omega=\frac{1+k^{2}}{2}
$$

f) $A(x)=A_{0} \operatorname{dn}(x, k)$

$$
A_{0}= \pm \frac{1}{k} \sqrt{\frac{V_{0 l}-k^{2}}{C_{1}^{2}+2 \sigma}}, \quad \omega=\frac{k^{2}}{2}-1+\frac{V_{0 l}}{k^{2}} .
$$

Using the above mapping we can readily construct the stationary solutions of the corresponding complex NLS with linear complex potentials given by

$$
W_{l}=\frac{3}{2} C_{1} A_{x}, \quad C_{1}=\frac{2}{3} \frac{W_{0 l}}{A_{0}}
$$


and with the phase given by $\theta(x)=C_{1} \int_{-\infty}^{x} A(y) \mathrm{d} y$. Thus, for example, from the solution a) we get

$$
\begin{aligned}
A & =A_{0} \operatorname{cn}(x, k), & V_{l}(x) & =V_{0 l} \operatorname{cn}^{2}(x, k) \\
A_{0} & =\frac{\sqrt{9\left(k^{2}+V_{0 l}\right)+2 W_{0 l}^{2}}}{3 \sqrt{|\sigma|}}, & \omega & =\frac{1-2 k^{2}}{2} \\
W_{l} & =-W_{0 l} \operatorname{sn}(x) \operatorname{dn}(x), & \theta(x) & =\frac{2 W_{0 l}}{3 k} \arccos (\operatorname{dn}(x)) .
\end{aligned}
$$

In similar manner one proceeds with the other solutions above. It is also clear that exact solutions of this type can be constructed also for other types of linear elliptic potentials (we omit them for brevity).

\subsection{Case $n=2$}

As a further application of the ansatz (8) we consider the case $n=2$ for which the mappings involves higher order nonlinearities. We assume as before that $V_{n l}=$ $W_{n l}=0$. In order to balance the quintic nonlinearity in equation (9), the potential $V_{l}$ must be taken as in equation (14). We take $\tilde{V}_{l}=V_{0 l} \mathrm{cn}{ }^{2}(x, k)$ and consider a solution of the form $A(x)=A_{0} \operatorname{cn}(x, k)$. One can then check that this is a solution of (9) with

$$
V_{l}(x)=V_{0 l} \mathrm{cn}^{2}(x, k)-\frac{C_{2}^{2}}{2} A_{0}^{4} \mathrm{cn}^{4}(x, k)
$$

if $A_{0}^{2}=V_{0 l}+k^{2}$ and $\omega=\frac{1-2 k^{2}}{2}$. From the mapping we then have that

$$
\begin{gathered}
C_{2}=\frac{W_{0 l}}{2 A_{0}^{2}}=\frac{W_{0 l}}{2\left(V_{0}+k^{2}\right)} \\
W_{l}(x)=2 C_{2} A A_{x}=-W_{0 l} \operatorname{cn}(x) \operatorname{sn}(x) \operatorname{dn}(x)
\end{gathered}
$$

and the phase is

$$
\theta(x)=x-\frac{x}{k^{2}}+\frac{1-k^{2}+k^{2} \mathrm{cn}^{2}(x, k)}{k^{2} \operatorname{dn}^{2}(x, k)} E(\operatorname{am}(x, k), k) .
$$

As a further example of $n=2$ we consider the case of pure nonlinear optical lattices, i.e., $V_{l}=W_{l}=0$. Fixing $\tilde{V}_{n l}=0$ and looking for solutions of the type $A(x)=A_{0} \operatorname{cn}(x, k)$, we have from equation (15) that that

$$
V_{n l}(x)=-\frac{C_{2}^{2}}{2} A_{0}^{2} \mathrm{cn}^{2}(x, k)
$$


with $C_{2}$ fixed according to equation (13) as $C_{2}=W_{0 n l} / 2$. One can easily check that this is indeed a solution of the eigenvalue problem (9) if

$$
\omega=\frac{1}{2}-k^{2}, \quad A_{0}=\frac{k}{\sqrt{|\sigma|}}
$$

(we consider $\sigma<0$ ). From the mapping we have that this is also a solution of the NLS with the complex part of the nonlinear potential fixed according to equation (10) as

$$
W_{n l}(x)=2 C_{2} \frac{A_{x}}{A}=-W_{0 n l} \frac{\operatorname{sn}(x, k) \operatorname{dn}(x, k)}{\operatorname{cn}(x, k)} .
$$

For the cases $n>2$ one can proceed in similar manner.

\subsection{General Case}

Let us now consider an example with the more general ansatz (16). To this regard we take $F(x)=\frac{1}{2}\left(C_{0}+C_{2} A^{2}\right) A^{2}$ and look for solutions of the form $A(x)=$ $A_{0} \operatorname{dn}(x, k)$. Let us fix the linear potentials as $V_{l}=W_{l}=0$ and the real nonlinear potential as $V_{n l}=V_{0 n l}-\alpha_{2}^{2} A^{2}$ with $V_{0 n l}$ a constant and with $\alpha_{n}=\frac{C_{n}}{\sqrt{2}}, n=0,2$ (notice that we fixed all coefficients for $n \neq 0,2$, equal to zero). By substituting these expressions into the real eigenvalue problem we find that $A(x)$ is indeed a solution if

$$
\omega=\alpha_{0}^{2}+\frac{k^{2}}{2}-1, \quad \alpha_{0}=-\frac{1+A_{0}^{2}\left(\sigma+V_{0 n l}\right)}{2 A_{0}^{2} \alpha_{2}} .
$$

Thus, for example, if we fix $V_{0 n l}=2 / k^{2}, \alpha_{2}=-1 / k$ and consider $\sigma=1$ (repulsive interactions), we have

$$
\left.V_{n l}=\frac{2-\mathrm{dn}^{2}(x, k)}{k^{2}}=\frac{1}{k^{2}}+\mathrm{sn}^{2}(x, k)\right) .
$$

From equation (36) we have

$$
\begin{aligned}
& \omega=\sigma-1+\frac{1}{A_{0}^{2}}+\frac{k^{2}\left[\left(1+2 \sigma A_{0}^{2}\right)+\left(\sigma^{2}+2\right) A_{0}^{4}\right]}{4 A_{0}^{4}}+\frac{1}{k^{2}} \\
& \alpha_{0}=\frac{1}{k}+\frac{k\left(1+\sigma A_{0}^{2}\right)}{2 A_{0}^{2}}
\end{aligned}
$$

and from (16) we get the function $F$ as

$$
F(x)=\frac{1}{\sqrt{2}}\left(\alpha_{0}+\alpha_{2} A^{2}\right) A^{2}
$$




$$
=\frac{A_{0}^{2} \mathrm{dn}^{2}(x, k)}{2 \sqrt{2} k}\left[2+\frac{k^{2}}{A_{0}^{2}}\left(1+\sigma A_{0}^{2}\right)-2 A_{0}^{2} \mathrm{dn}^{2}(x, k)\right] .
$$

Substituting into equations (18) we finally get the complex potential as

$$
W_{n l}=\sqrt{2} k \frac{\operatorname{sn}(x, k) \operatorname{cn}(x, k)}{\operatorname{dn}^{3}(x, k)} \times\left[2-\frac{1}{A_{0}^{2}}-\frac{k^{2}}{2 A_{0}^{4}}\left(1+\sigma A_{0}^{2}\right)-2 k^{2} \operatorname{sn}^{2}(x, k)\right] .
$$

The phase of the solution can be readily obtained from equation (19). Notice that in the case $\sigma=1, A_{0}=1$, this solution coincides with the one derived in [2] with a slightly different approach. We remark that the above solutions of the complex NLS equations not only have real energies but are also stable (not shown for brevity) under time evolution.

\section{Conclusions}

In conclusion we have demonstrated the possibility to construct stationary solutions of the linear and nonlinear Schrodinger equation with complex potentials via a mapping with stationary solutions of the NLS equation with suitable real potentials. In particular we showed that by means of this mapping it is possible to construct sets of exact solutions with real energies for different types of complex potentials. The presented approach can be applied to other types equations, including the linear Schrödinger equation describing quantum dissipative oscillators, and the NLS equation with arbitrary higher order nonlinearities, as it will be discussed elsewhere.

\section{Acknowledgements}

It is a pleasure to dedicate this paper to Professor Vladimir Gerdjikov on the occasion of his 65th birthday in 2012. Partial support from the Ministero dell' Istruzione, dell' Università e della Ricerca (MIUR) through a Programma di Ricerca Scientifica di Rilevante Interesse Nazionale (PRIN) 2010-2011 initiative, is acknowledged.

\section{References}

[1] Abdullaev F., Gammal A., da Luz H. and Tomio L., Dissipative Dynamics of Matter-Wave Solitons in a Nonlinear Optical Lattice, Phys. Rev. A 76 (2007) 043611-1-9. 
[2] Abdullaev F., Konotop V., Salerno M. and Yulin A., Dissipative Periodic Waves, Solitons, and Breathers of the Nonlinear Schrödinger Equation with Complex Potentials, Phys. Rev. E 82 (2010) 056606-1-6.

[3] Abdullaev F. and Salerno M., Gap-Townes Solitons and Localized Excitations in Low-Dimensional Bose-Einstein Condensates in Optical Lattices, Phys. Rev. A 72 (2005) 033617-1-12.

[4] Akhmediev N. and Ankiewicz A. (Eds), Dissipative Solitons, Lecture Notes in Physics 661, Springer, Berlin 2005.

[5] Aranson I. and Kramer L., The World of the Complex Ginzburg-Landau Equation, Rev. Mod. Phys. 74 (2002) 99-143.

[6] Bender C., Boettcher S., Real Spectra in Non-Hermitian Hamiltonians Having PT Symmetry, Phys. Rev. Lett. 80 (1998) 5243-5246.

[7] Bludov Yu. and Konotop V., Nonlinear Patterns in Bose-Einstein Condensates in Dissipative Optical Lattices, Phys. Rev. A 81 (2010) 013625-1-8.

[8] Brazhnyi V. and Konotop V., Theory of Nonlinear Matter Waves in Optical Lattices, Mod. Phys. Lett. B 18 (2004) 627-651.

[9] Guo A., Salamo G., Duchesne D., Morandotti R., Volatier-Ravat M., Aimez V., Siviloglou G., and Christodoulides D., Observation of PT-Symmetry Breaking in Complex Optical Potentials, Phys. Rev. Lett. 103 (2009) 093902$1-4$.

[10] See e.g. the special issue of \# 32: The Physics Non-Hermitian Operators, J. Phys. A 39 (2006)

[11] Longhi S., Bloch Oscillations in Complex Crystals with PT Symmetry, Phys. Rev. Lett. 103 (2009) 123601-1-4.

[12] Muga J., Palao J., Navarro B. and Egusquiza I., Complex Absorbing Potentials, Phys. Rep. 395 (2004) 357-426.

[13] Musslimani Z., Markis K., El-Ganainy R. and Christodoulides D., Optical Solitons in PT Periodic Potentials, Phys. Rev. Lett. 100 (2008) 030402-1-4.

[14] Musslimani Z., Markis K., El-Ganainy R. and Christodoulides D., Analytical Solutions to a Class of Nonlinear Schrödinger Equations with PT-Like Potentials, J. Phys. A 41 (2008) 244019.

[15] Reuter C., Makris K., El-Ganainy R., Christodoulides D., Segev M. and Kip D., Observation of Parity-Time Symmetry in Optics, Nature Phys. 6 (2010) 192-195.

[16] Salerno M., Laser Physics, Macroscopic Bound States and the Josephson Effect in Bose-Einstein Condensates in Optical Lattices 14 (2005) 620-625.

[17] Schindler J., Li A., Zheng M., Ellis F. and Kottos T., Experimental Study of Active LRC Circuits with PT Symmetries, Phys. Rev. A 84 (2011) 040101-1-5. 
[18] Staliunas K., Herrero R. and Vilaseca R., Subdiffraction and Spatial Filtering due to Periodic Spatial Modulation of the Gain-Loss Profile, Phys. Rev. A 80 (2009) 013821-1-6.

Mario Salerno

Dipartimento di Fisica "E.R. Caianiello" and INFN

Sezione di Napoli-Gruppo Collegato di Salerno

Università di Salerno

via Giovanni Paolo II, Stecca 8-9

84084 Fisciano (SA), ITALY

E-mail address: salerno@sa.infn.it 